\title{
New Omega-3 Derivatives Reduce Airway Inflammation and Prevent Rho- Kinase Activation in an Allergic Model of Asthma
}

Caroline Morin $^{1,2}$, Samuel Fortin ${ }^{1}$ and Éric Rousseau ${ }^{2 \star}$

${ }^{1}$ SCF Pharma, Ste-Luce, Quebec, Canada

${ }^{2}$ Department of Physiology and Biophysics, Faculty of Medicine and Health Sciences, Université de Sherbrooke, Sherbrooke, Quebec, Canada

\begin{abstract}
Asthma is a chronic inflammatory airways disease characterized by early and late asthmatic reactions that are associated with infiltration and activation of inflammatory cells in the airways. Bronchial hyperresponsiveness to a variety of stimuli is part of the symptomology which include neurotransmitters and inflammatory mediators. Omega-3 fatty acids such as docosahexaenoic acid (DHA) and eicosapentaenoic acid (EPA) are known to reduce inflammation in the lung. In the present study, we synthesized new omega-3 polyunsaturated fatty acid (n3-PUFA) derivatives, namely DHA monoglyceride (MAG-DHA) and EPA monoglyceride (MAG-EPA), and investigated their effects on lung inflammation and RhoA activation in an in vivo guinea pig model of allergic asthma. Histological analyses and leukocyte count in bronchoalveolar lavages revealed that oral MAG-DHA and MAG-EPA treatments led to less inflammatory cell recruitment in the lung of ovalbumine (OVA)-sensitized guinea pigs when compared to lung from control animals. Results also revealed a reduction in mucus production in OVA-sensitized animals treated with either MAG-DHA or MAG-EPA. Moreover, per os n-3 PUFA administrations prevented NFKB activation and COX2 over-expression in OVA-sensitized animals. Mechanical tension measurements revealed that oral MAG-DHA and MAG-EPA treatments prevented U-46619-induced bronchial hyperresponsiveness in OVA-sensitized guinea pigs. MAG-DHA and MAG-EPA also prevented U-46619-induced $\mathrm{Ca}^{2+}$ hypersensitivity of bronchial smooth muscle through a decrease in RhoA activation. Together, these findings provide key evidences regarding the mode of action of MAG-DHA and MAG-EPA in the lung and point to new potential therapeutic strategies for modulating inflammation and airway hyperresponsiveness in asthma.
\end{abstract}

Keywords: Airway hyperresponsiveness; Asthma; DHA; EPA; Inflammation; RhoA

\section{Introduction}

Asthma is a chronic disease characterized by airways hyperresponsiveness, inflammation, remodelling of the media and reversible bronchial obstruction. Airway structural cells, recruited inflammatory cells as well as numerous mediators such as cytokines, chemokines and adhesion molecules are involved in the pathogenesis of asthma [1,2]. Despite the availability of several classes of asthma medications such as beta-agonists, leukotriene antagonists and corticosteroids, nearly $50 \%$ of asthmatics fail to benefit from one or more of these drugs. Moreover, none of the treatments are preventive or curative such that the disease has reached epidemic proportions worldwide and its incidence continuously increasing, especially in developing countries [3].

Airway hyperresponsiveness (AHR) considered as the hallmark of the asthma phenotype, is defined as the ease with which the airways narrow in response to a bronchoconstrictive challenge and manifests as a combination of increased sensitivity and reactivity for a given stimulus [4]. Several mechanisms have been suggested to explain AHR, such as alterations in neural control of airway smooth muscles (ASM) [4], inflammatory process resulting in the release of cytokines and lipid mediators [3] and abnormal calcium handling by ASM cells [5,6]. The contraction of smooth muscles including airway smooth muscles is mediated by both $\mathrm{Ca}^{2+}$-dependent and $\mathrm{Ca}^{2+}$-independent pathways. The latter independent pathway, termed $\mathrm{Ca}^{2+}$ sensitization, is mainly regulated by a small monomeric GTP-binding protein, RhoA, and its downstream target Rho-kinase. Inactive RhoA exists in the cytosol with its partner molecule, GDP dissociation inhibitor (RhoGDI). G protein-coupled receptors, upon binding their respective ligands, activate the heterotrimeric $\mathrm{G}_{12 / 13}$ protein [7], which in turn triggers one or more tyrosine kinases and other signaling molecules, culminating in the activation of a Rho-specific guanine nucleotide exchange factor (RhoGEF). These signaling molecules displace RhoGDI and stimulate the exchange of GDP for GTP. Once activated, RhoA translocates to the membrane and activates Rho associated kinase (ROCK), which in turn targets myosin light chain phosphatase (MLCP); phosphorylation of the constitutively active MLCP at its myosin binding region leads to its inhibition, favoring a larger quantity of phosphorylated myosin at lower levels of $\left[\mathrm{Ca}^{2+}\right] \mathrm{i}$, a phenomenon termed $\mathrm{Ca}^{2+}$ sensitization. Following deactivation of the receptor, RhoA inactivates by hydrolyzing GTP and reassociates with RhoGDI [7]. In animal models of allergic bronchial asthma, an augmented agonist-induced and RhoA-mediated contraction of bronchial smooth muscle has been demonstrated [8]. Moreover, the specific Rho-kinase inhibitor (Y-27632) has been shown to suppress AHR in mice repeatedly challenged with ovalbumin sensitization [9]. Hence, changes in contraction mediated by the Rho/ Rho-kinase pathway upon allergen sensitization could contribute to the development of AHR under these conditions.

Clinical assessment of dietary supplementation of omega-3 (n-3) polyunsaturated fatty acids (PUFA), including eicosapentaenoic acid

*Corresponding author: Éric Rousseau, PhD, Department of Physiology and Biophysics, Faculty of Medicine and Health Sciences, Université de Sherbrooke 3001, $12^{\mathrm{e}}$ Avenue Nord, Sherbrooke, J1H 5N4, QC, Canada, Tel: (819) 564-5306; Fax: (819) 564-5399; E-mail: Eric.Rousseau@USherbrooke.ca

Received November 26, 2011; Accepted January 18, 2012; Published January 22, 2012

Citation: Morin C, Fortin S, Rousseau É (2012) New Omega-3 Derivatives Reduce Airway Inflammation and Prevent Rho-Kinase Activation in an Allergic Model of Asthma. J Aller Ther S1:003. doi:10.4172/2155-6121.S1-003

Copyright: (C) 2012 Morin C, et al. This is an open-access article distributed unde the terms of the Creative Commons Attribution License, which permits unrestricted use, distribution, and reproduction in any medium, provided the original author and source are credited. 
(EPA) and docosahexaenoic acid (DHA), indicates their beneficial impact in a wide range of human diseases in which unresolved inflammation is suspected to be a key component in disease pathogenesis [10,11]. Several putative mechanisms have been proposed to explain the benefits of n-3 PUFAs: (i) via substrate competition, preventing conversion of arachidonic acid into pro-inflammatory eicosanoids such as prostaglandins (PGs) and leukotrienes (LTs); (ii) serving as an alternative substrate to produce less potent 5 -series LTs and 3-series PGs and thromboxanes [12-14]. Systematic metabolomic studies of lipid mediators in the course of acute inflammatory responses have revealed that n-3 PUFA-derived mediators are produced within inflammatory exudates, including E-series resolvins such as resolvin E1 from EPA and D-series resolvins and protectin D1 from DHA [15-17]. These newly identified chemical mediators appear to exert potent antiinflammatory and pro-resolving actions both in vitro and in vivo [18].

Asthma is directly associated with an increase levels in omega-6 (n6) fatty acid levels, such as arachidonic acid, in blood circulation and tissues [11]. Other studies have reported that the physiological levels of omega-3 fatty acid derivatives are relatively low in blood circulation, suggesting the importance of omega-3 supplementation [10,16,19-21]. Thus, new EPA and DHA sn1-monoacylglycerides were synthesized. These compounds are non-toxic and their metabolites are found in blood circulation and lung tissues [22-24]. The aim of the present study was to determine the ability of MAG-DHA and MAG-EPA to reduce inflammation and AHR using an in vivo model of allergic asthma. Herein we report the first evidence that MAG-DHA and MAG-EPA display anti-inflammatory properties and prevent RhoA activation leading to AHR in lung tissues.

\section{Materials and Methods}

\section{Synthesis of omega-3 monoglyceride}

MAG-EPA and MAG-DHA were synthesized as previously described using either ethyl docosahexaenoate or ethyl eicosapentaenoate as starting material [22].
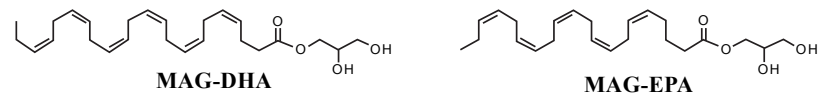

\section{Sensitization and challenge protocols}

Male or female albino guinea pigs, (Hartley 200-250 g) were obtained from Charles Rivers Laboratories (Montreal, Qc, Canada). All procedures involving animal tissues were performed according to current Canadian Council for Animal Care (CCAC) guidelines. Guinea pigs were sensitized with i.p. injections of $10 \mu \mathrm{g}$ ovalbumin (OVA) (Grade V, Sigma Chemical, St Louis, MO) plus $1 \mathrm{mg}$ of $\mathrm{Al}(\mathrm{OH})_{3}$ as an adjuvant in $0.4 \mathrm{ml}$ PBS, on day 0 and 7 . Controls animals received PBS by i.p. injection. MAG-DHA or MAG-EPA were administrated per os (231 mg/kg, according to Health Canada Draft Guidelines) [25] on day 0 and 7, 30 minutes before OVA sensitization. On days 14 to 18 , the OVA-sensitized guinea pigs received MAG-DHA or MAG-EPA 30 minutes before an aerosol challenge containing 1.5\% OVA for $10 \mathrm{~min}$ or until suffocation. Control animals were challenged with aerosolized PBS. The aerosols were generated with a small particle aerosol generator model SPAG-2 (ICN, Montreal, QC, Canada). On day 19, 24 hours after the last aerosol challenge, the OVA-sensitized guinea pigs, either untreated or treated with either MAG-DHA or MAGEPA, were anesthetized, the bronchi removed and used immediately for isometric tension measurements while lung tissues were harvested for histological analysis or homogenate preparation for Western Blot experiments. Bilateral bronchoalveolar lavages (BAL) (2 aliquots of 2 $\mathrm{ml}$ of PBS plus $0.6 \mathrm{mM}$ EDTA) were performed on control animals and on OVA-sensitized guinea pigs untreated or treated with either MAGDHA or MAG-EPA $(231 \mathrm{mg} / \mathrm{kg})$. No BAL or histological analysis was performed on animals undergoing tension measurement or lung homogenate preparations. Moreover, no behavioral or physical signs of toxicity with MAG-DHA and MAG-EPA treatments were observed. The illustration below summarizes the sensitization protocol as a function of time:

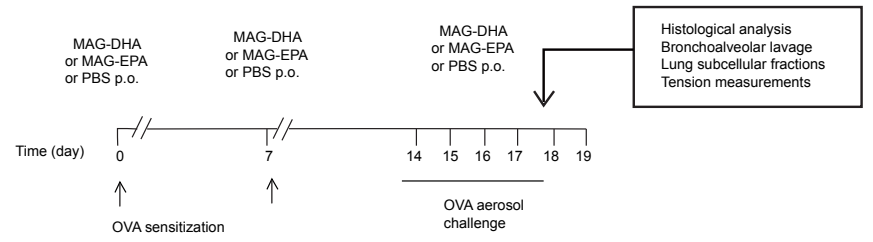

\section{Allergen-initiated lung inflammation}

Guinea pig lungs were fixed in $10 \%$ buffered formalin and paraffin embedded after which thin sections $(3 \mu \mathrm{m})$ were stained with hematoxylin-eosin and Alcian blue according to standard protocols. Measurement of inflammatory mediator was determined in cell-free BAL fluid (2000g, $10 \mathrm{~min}$ ) by sensitive and specific ELISA, for TNFo (Cusabio Biotech, Newark, DE). Cells in BAL fluids were resuspended in PBS, enumerated by hemocytometer, and concentrated onto microscope slides by cytocentrifugation at $265 \mathrm{~g}$. Cells were stained with a Wright-Giemsa stain (Sigma-Aldrich) to determine leukocyte differential counts [23].

\section{Western blot analysis}

Lung homogenates were prepared from control as well as OVAsensitized animals untreated or treated with either MAG-DHA or MAG-EPA. Lung tissues were removed, weighed and promptly transferred in a buffer containing $0.3 \mathrm{M}$ sucrose, $20 \mathrm{mM}$ K-PIPES, $4 \mathrm{mM}$ EGTA and a cocktail of protease and phosphatase inhibitors (Protease-inhibitor pellets from Roche Diagnostics, Indianapolis, IN, USA, $+10 \mu \mathrm{M} \mathrm{Na} \mathrm{VO}_{3}$ ). Tissues were then homogenized on ice, frozen in liquid nitrogen and stored at $-80^{\circ} \mathrm{C}$. Samples containing equal amounts of protein were separated on SDS-polyacrylamide gels, and the proteins subsequently transferred by electroblotting onto nitrocellulose membranes (Bio-Rad, Mississauga, ON, Canada). Western blots were performed using specific antibodies against COX2, I $\mathrm{B} \alpha, \mathrm{p} 65-\mathrm{NF} \kappa \mathrm{B}$, its phosphorylated form (Anti P-NFKB) and $\beta$-actin proteins [23]. Membranes were incubated for $1 \mathrm{~h}$ at $23^{\circ} \mathrm{C}$ with peroxidase-conjugated donkey anti-rabbit IgG1 antiserum (Amersham, QC, Can) and revealed by Enhanced Chemiluminescence (Roche, QC, Can). Blot immunostainings were digitized and analyzed with Lab-Image software 2.7-2.

\section{Isometric tension measurements}

The mechanical effects induced by U46619 (a thromboxane A2 analog) were measured as previously described [23,26]. Briefly, bronchial rings were mounted in isolated organ baths (Radnoti Glass Tech., Monrovia, CA), containing $6 \mathrm{ml}$ of Krebs solution at $37^{\circ} \mathrm{C}$, bubbled continuously with a $95 \% \mathrm{O}_{2} / 5 \% \mathrm{CO}_{2}$ mixture and to which an initial load of $0.8 \mathrm{~g}$ was applied. Tissues were allowed to equilibrate for $1 \mathrm{~h}$ in Krebs solution and washed out every $15 \mathrm{~min}$. Passive and active tensions were assessed using transducer systems coupled to Polyview software (Grass-Astro-Med Inc, West Warwick, RI) for facilitating data acquisition and analysis. The measurement of resulting induced myofilament $\mathrm{Ca}^{2+}$ sensitivity was performed as previously reported 
Citation: Morin C, Fortin S, Rousseau É (2012) New Omega-3 Derivatives Reduce Airway Inflammation and Prevent Rho-Kinase Activation in an Allergic Model of Asthma. J Aller Ther S1:003. doi:10.4172/2155-6121.S1-003

Page 3 of 9

by using a $\beta$-escin permeabilization protocol on guinea pig bronchi $[23,26]$.

\section{Measurement of RhoA activity}

RhoA activity was assessed in homogenized lung tissue samples by pulldown assays using the Rho-binding domain of the Rho effector: Rhotekin, according to the manufacturer's instructions (RhoA activation assay kit, Cell Biolabs, Cedarlane laboratories, Burlington, ON, Canada) [27].

\section{Data analysis and statistics}

Results are expressed as means \pm S.E.M. with $\mathrm{n}$ indicating the number of experiments. Statistical analyses were performed using a Student $t$ test or a one-way analysis of variance (ANOVA). Differences were considered statistically significant when $p<0.05$. Data curve fittings were performed using Sigma Plot 11 (SPSS-Science, Chicago, IL) to determine $\mathrm{EC}_{50}$ values [23].

\section{Results}

Effect of oral MAG-DHA and MAG-EPA administration on allergen-induced lung inflammation

An in vivo model of allergic asthma was used to determine the impact of MAG-DHA and MAG-EPA on lung inflammation and AHR in OVA-sensitized guinea pigs. The n-3 PUFA monoacylglycerides
A

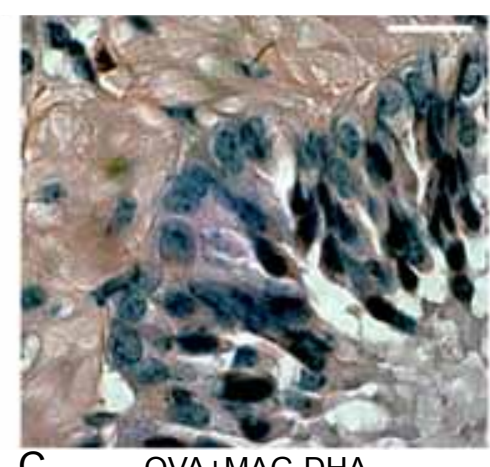

C OVA+MAG-DHA

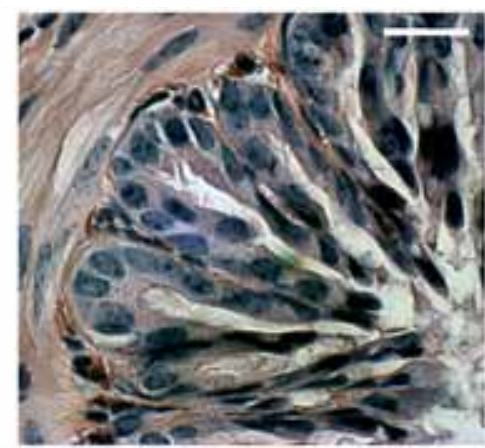

$\mathbf{E}$

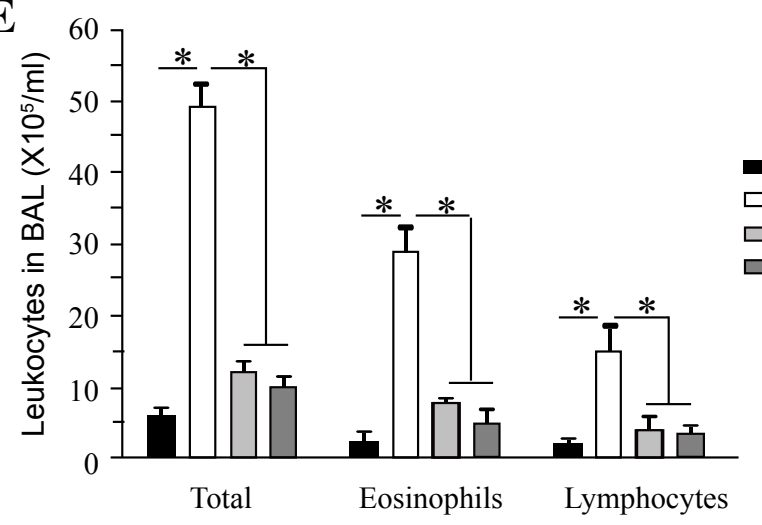

B OVA

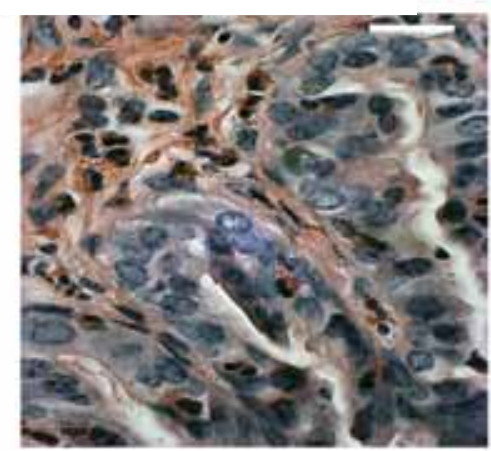

D OVA+MAG-EPA

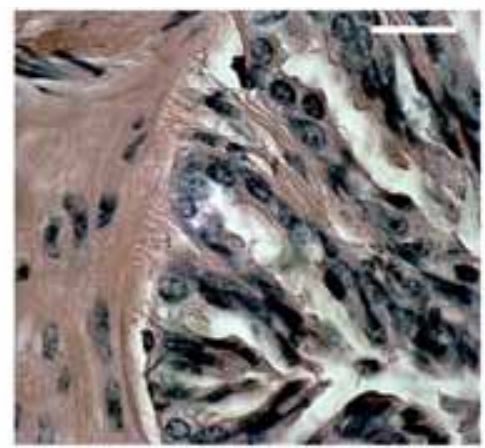

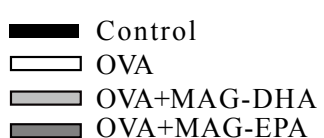
OVA+MAG-EPA

Figure 1: MAG-DHA and MAG-EPA reduce airway inflammation in ovalbumin (OVA)-sensitized lung tissue.

A-D: Representative lung tissue sections obtained from a non-sensitized guinea pig (control) (A) as well as from OVA-sensitized (B), OVA-sensitized + MAG-DHAtreated $(\mathbf{C})$ and OVA-sensitized + MAG-EPA-treated animals (D). The degree of inflammation was assessed by determining the extent of inflammatory infiltrates present in lung sections stained with Hematoxylin-Eosin. Scale bar $40 \mu \mathrm{m}$. Images are representative of $n=8,10,11$ and 8 (animals, experiments) per group, respectively. E: Total leukocytes, eosinophils (Eos.) and lymphocytes (Lymph.) in BAL fluid were enumerated and identified after Wright-Giemsa staining. Results are expressed as mean \pm SEM, $\left(n=7\right.$ per group), ${ }^{*} P<0.05$. 
Citation: Morin C, Fortin S, Rousseau É (2012) New Omega-3 Derivatives Reduce Airway Inflammation and Prevent Rho-Kinase Activation in an Allergic Model of Asthma. J Aller Ther S1:003. doi:10.4172/2155-6121.S1-003

were administrated per os at pharmacological dose $(231 \mathrm{mg} / \mathrm{kg})$ [25]. The extent of inflammation in lung tissues was determined in control and OVA-sensitized guinea pigs treated or not with MAG-DHA and MAG-EPA. Histological analyses performed on lung sections stained with hematoxylin-eosin revealed that animals receiving either MAGDHA or MAG-EPA had substantially less eosinophils and lymphocytes in the peribronchial regions and airspaces compared to OVA-sensitized animals (Figure 1 A-D). In BAL fluids, MAG-DHA and MAG-EPA treatments led to fewer total leukocytes, namely eosinophils and lymphocytes, when compared to the number of leucocytes found in BAL fluids of untreated OVA-sensitized guinea pigs (Figure 1E)

\section{Effect of oral MAG-DHA and MAG-EPA treatments on} mucus production

To determine the effects of per os n-3 PUFA monoglyceride treatments on airway mucus production, Alcian blue staining was performed on lung tissue sections derived from control and OVAsensitized guinea pigs treated or not with either MAG-DHA or MAGEPA. Histological analyses revealed that MAG-DHA and MAG-EPA reduced goblet cell hyperplasia and airway mucus production in guinea pig lung when compared to OVA-sensitized animals (Figure 2 A-D). Quantitative analysis of lung stained tissue sections derived from OVAsensitized animals revealed a 2.2 fold increase when compared to the levels obtained in sections derived from non-sensitized control guinea pigs (Figure 2E). Image analyses also revealed that in lung tissue sections derived from OVA-sensitized animals treated with either MAG-DHA or MAG-EPA, significant reductions of 53 and $57 \%$ were observed when compared to the levels obtained in sections derived from OVAsensitized guinea pigs (Figure 2E). Moreover, no significant difference was quantified between n-3 PUFA-treated and control animals (Figure $2 \mathrm{E}$, grey versus dark bar columns).
A

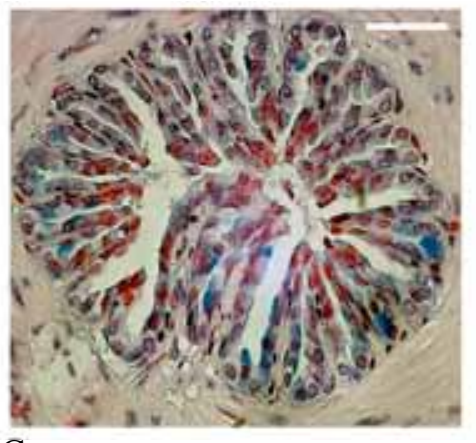

C

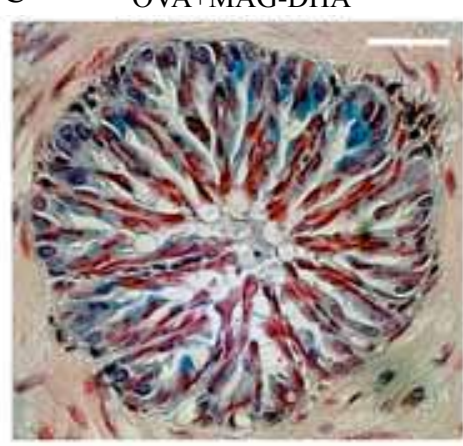

B

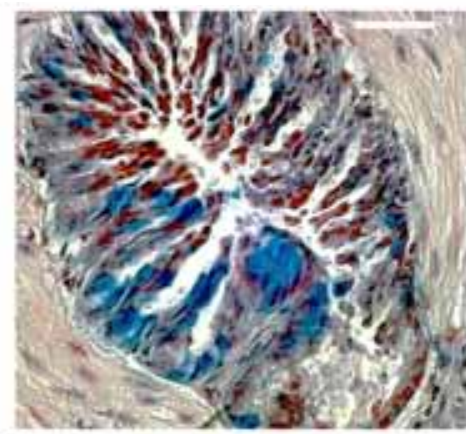

D OVA+MAG-EPA

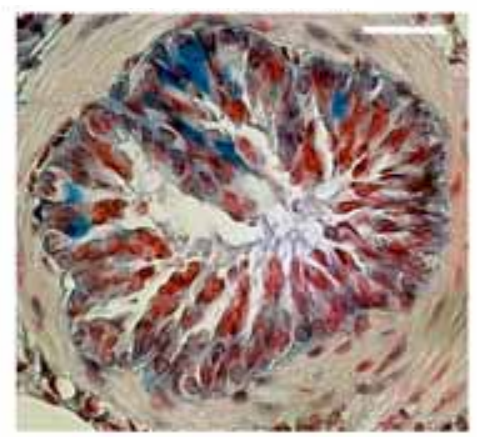

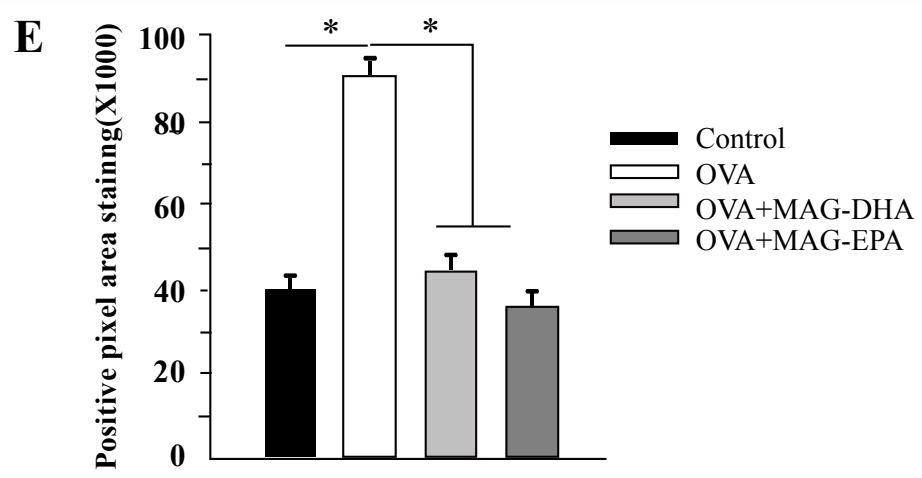

Figure 2: MAG-DHA and MAG-EPA reduce mucus production in OVA-sensitized lung tissue. Lung tissue sections obtained from a non-sensitized guinea pig Control (A), as well as from OVA-sensitized (B), OVA-sensitized + MAG-DHA-treated (C) and OVA-sensitized + MAG-EPA-treated (D) animals were stained with Alcian blue. Mucus secretion and goblet cells containing mucus are stained in blue. Scale bar $40 \mu \mathrm{m}$. Images are representative of $n=8,10,11$ and 8 (animals, experiments) per group, respectively $(\mathbf{E})$ : Quantitative analysis displaying the mean immuno-positive pixel area staining of Alcian blue from lung tissue sections $(n=8-10-11-8$ per group, $\left.{ }^{*} \mathrm{P}<0.05\right)$. 
Citation: Morin C, Fortin S, Rousseau É (2012) New Omega-3 Derivatives Reduce Airway Inflammation and Prevent Rho-Kinase Activation in an Allergic Model of Asthma. J Aller Ther S1:003. doi:10.4172/2155-6121.S1-003

Page 5 of 9

\section{Effect of MAG-DHA and MAG-EPA treatments on NFKB pathway}

To determine whether n-3 PUFA monoglycerides reduce allergeninduced airway inflammation, the activation of nuclear factor kappa $\mathrm{B}$ $(\mathrm{NF \kappa B})$ was investigated. Activation of this factor is usually correlated with phosphorylation of the p65 NFKB subunit as well as a reduction in I $\mathrm{B} \alpha$ due to extensive ubiquitination and proteosomal degradation of this inhibitory subunit, which results in an increased nuclear translocation of the p65 NFKB subunit [28]. Western blot analysis revealed that OVA-sensitized animals resulted in $\mathrm{I} \kappa \mathrm{B} \alpha$ degradation and an increased phosphorylation of p65 subunit staining in lung homogenate fractions when compared to preparations derived from control guinea pigs (Figure 3A). However, MAG-DHA and MAGEPA treatments prevented the degradation of $I \kappa B \alpha$ and concomitant phosphorylation of $\mathrm{p} 65 \mathrm{NF \kappa B}$ subunit induced by the allergen when compared to OVA-sensitized guinea pigs. Since increased COX2 activity and expression is related to an inflammatory status in several tissues and that OVA sensitization is able to increase COX2 expression in guinea pig lung $[23,29]$, experiments were therefore designed to evaluate the expression level of COX2 in control and OVA-sensitized guinea pigs treated or not with either MAG-DHA or MAG-EPA. OVA sensitization induced an increased staining level of COX2 protein detection when compared to levels obtained from control animals, whereas MAG-DHA and MAG-EPA prevented the over-expression of COX2 protein induced by OVA in guinea pig lung (Figure $3 \mathrm{~B}$ ).

\section{Effect of n-3 PUFA monoglyceride treatments on OVA- induced AHR}

The involvement of the Rho-kinase pathway was assessed using $\mathrm{U}-46619$, a thromboxane A2 analog known to activate the Rhokinase pathway and to induce airway smooth muscle tone. In order to determine the impact of MAG-DHA and MAG-EPA treatments on U-46619-induced tension in OVA-sensitized guinea pigs, experiments were designed to assess the mechanical properties of bronchial smooth muscle to cumulative concentrations of this pharmacological agonist. Figure 4 displays the cumulative concentration response curves (CCRC) to U-46619 in bronchial preparations derived from control, OVA-sensitized, OVA-sensitized + MAG-DHA-treated, as well as from OVA-sensitized + MAG-EPA-treated animals. Data revealed an overreactivity of the bronchi from OVA-sensitized guinea pigs to U-46619 with an $\mathrm{EC}_{50}$ value of $20 \mathrm{nM}$, whereas oral MAG-DHA and MAG-EPA administrations prevented the development of AHR to this agonist, with $\mathrm{EC}_{50}$ values of 54 and $49 \mathrm{nM}$, respectively (Figure 4). No difference was quantified between n-3 PUFA-treated animals and control guinea pigs receiving PBS only.

\section{Effects of n-3 PUFA monoglycerides on U-46619 induced $\mathrm{Ca}^{2+}$ hypersensitivity and RhoA activation in an OVA-sensitized model of asthma}

To assess the effect of MAG-DHA and MAG-EPA treatments on $\mathrm{U}-46619$ induced $\mathrm{Ca}^{2+}$ sensitivity, comparative analyses were performed on $\beta$-escin-permeabilized guinea pig bronchial rings. Figure
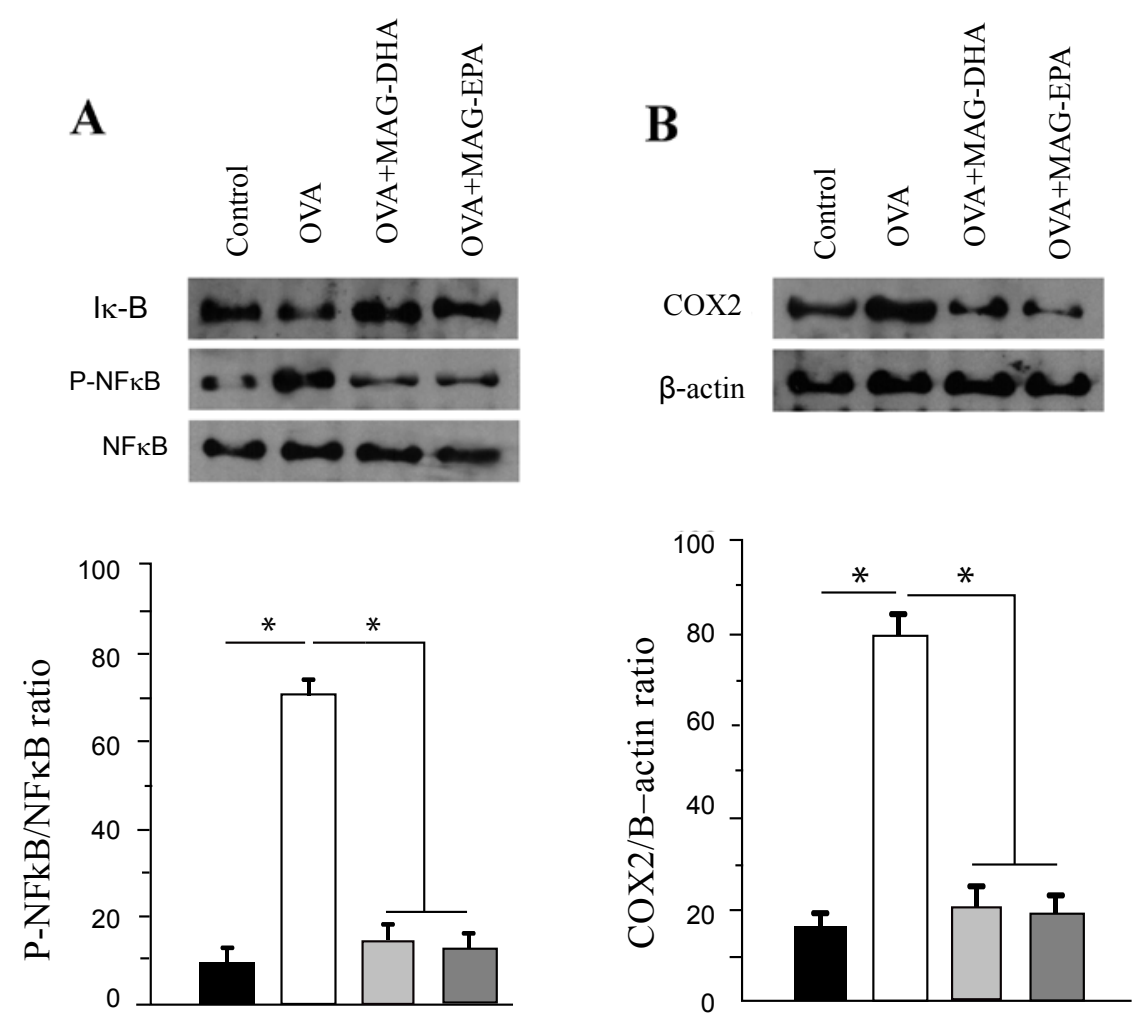

Figure 3: Effect of MAG-DHA and MAG-EPA on airway inflammatory markers in lung tissue. A: Western blot and quantitative analysis of lung homogenate fractions derived from Control, OVA, OVA + MAG-DHA and OVA + MAG-EPA, using specific antibodies against I $\mathrm{k} \alpha, \mathrm{p} 65 \mathrm{NF} \kappa \mathrm{B}$ and its phosphorylated form ( $\mathrm{n}=6$ ). B. Western blot and quantitative analysis of COX2 protein detection. Staining densities in the homogenates were expressed as a function of $\beta$-actin signals ( $=6$ ). ${ }^{*} \mathrm{P}<0.05$. 
Citation: Morin C, Fortin S, Rousseau É (2012) New Omega-3 Derivatives Reduce Airway Inflammation and Prevent Rho-Kinase Activation in an Allergic Model of Asthma. J Aller Ther S1:003. doi:10.4172/2155-6121.S1-003

Page 6 of 9

$5 \mathrm{~A}$ illustrates CCRC to free $\mathrm{Ca}^{2+}$ concentrations in the presence of 20 nM U-46619 on permeabilized bronchial rings obtained from control and OVA-sensitized tissues. When compared to control conditions (closed circles), an enhanced $\mathrm{Ca}^{2+}$ sensitivity to pre-calibrated $\mathrm{Ca}^{2+}$ step increases in the presence of U-46619 was observed in OVAsensitized bronchial tissues (Figure 5A, open triangles). However, MAG-DHA and MAG-EPA treatments resulted in a marked inhibitory effect on $\mathrm{Ca}^{2+}$-sensitivity to U-46619 (right shift) developed by OVAsensitized bronchi. Data analysis demonstrated that MAG-DHA and MAG-EPA treatments induced a shift in $\mathrm{EC}_{50}$ values $(1.54 \pm 0.06 \mu \mathrm{M}$ and $1.49 \pm 0.05 \mu \mathrm{M}$, respectively) toward higher $\mathrm{Ca}^{2+}$ concentrations when compared to OVA-sensitized untreated tissues challenged with $\mathrm{U}-46619(0.38 \pm 0.05 \mu \mathrm{M})$ (Figure 5A). However, the difference in $\mathrm{Ca}^{2+}$ sensitivity between control bronchi $\left(\mathrm{EC}_{50}\right.$ values of $\left.1.54 \pm 0.05 \mu \mathrm{M}\right)$ and tissues treated with either MAG-DHA or MAG-EPA was not significant (Figure 5A). In order to further investigate the putative processes that would support this negative feedback mechanism induced by n-3 PUFA monoglycerides on the $\mathrm{Ca}^{2+}$-tension relationship induced by $\mathrm{U}-46619$, experiments were performed to assess the activity of RhoA in lung homogenates derived from control and the 3 series of OVA-sensitized animals. RhoA activation assay was performed in homogenized tissue samples by pulldown assays using Rhotekin, the Rho-binding domain of the Rho effector. Data analysis revealed that oral MAG-DHA and MAGEPA treatments prevented the activation of RhoA, as demonstrated by a lower amount of RhoA coupled to GTP (Figure 5B), thus correlating with the functional measurements described above (Figure 4 and $5 \mathrm{~A}$ ).

\section{Discussion}

\section{Anti-inflammatory effects of n-3 PUFA monoacylglyceride derivatives}

Asthma prevalence continues to increase in North America despite advancements in treatment options for this pathology $[1,3]$. Alternative preventive treatment aimed at reducing the dose requirements of pharmacological interventions would henceforth be beneficial, in

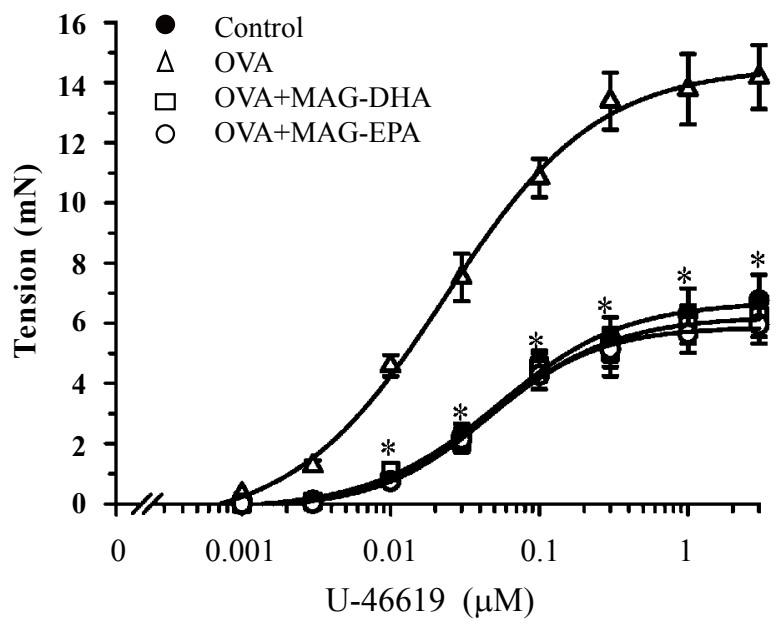

Figure 4: Effect of MAG-DHA and MAG-EPA treatments on airway hyperresponsiveness induced by $\mathrm{U}-46619$ in ovalbumin-sensitized guinea pigs. Cumulative concentration response curves (CCRC) to U-46619 generated in Control (closed circles), OVA sensitized (open triangles), OVA-sensitized + MAG-DHA-treated (open squares) and OVA-sensitized + MAG-EPA-treated (open circles) guinea pigs. Each point represents the mean \pm SEM, with $n=15$ for each experimental condition. ${ }^{*} \mathrm{P}<0.05$. addition to potentially reducing the public health and socioeconomic burden of this disease [1]. Over the past three decades, there has been significant interest in the therapeutic potential of fish oils rich in $n$-3 PUFA, such as DHA and EPA, for various inflammatory conditions such as asthma, rheumatoid arthritis and inflammatory bowel diseases $[11,21,30]$. Of interest, DHA levels in the respiratory tract are decreased in asthma as well as in other excess airway inflammation diseases, such as cystic fibrosis [19]. Epidemiological studies suggest that a diet enriched in marine fatty acids (fish oil) may have beneficial
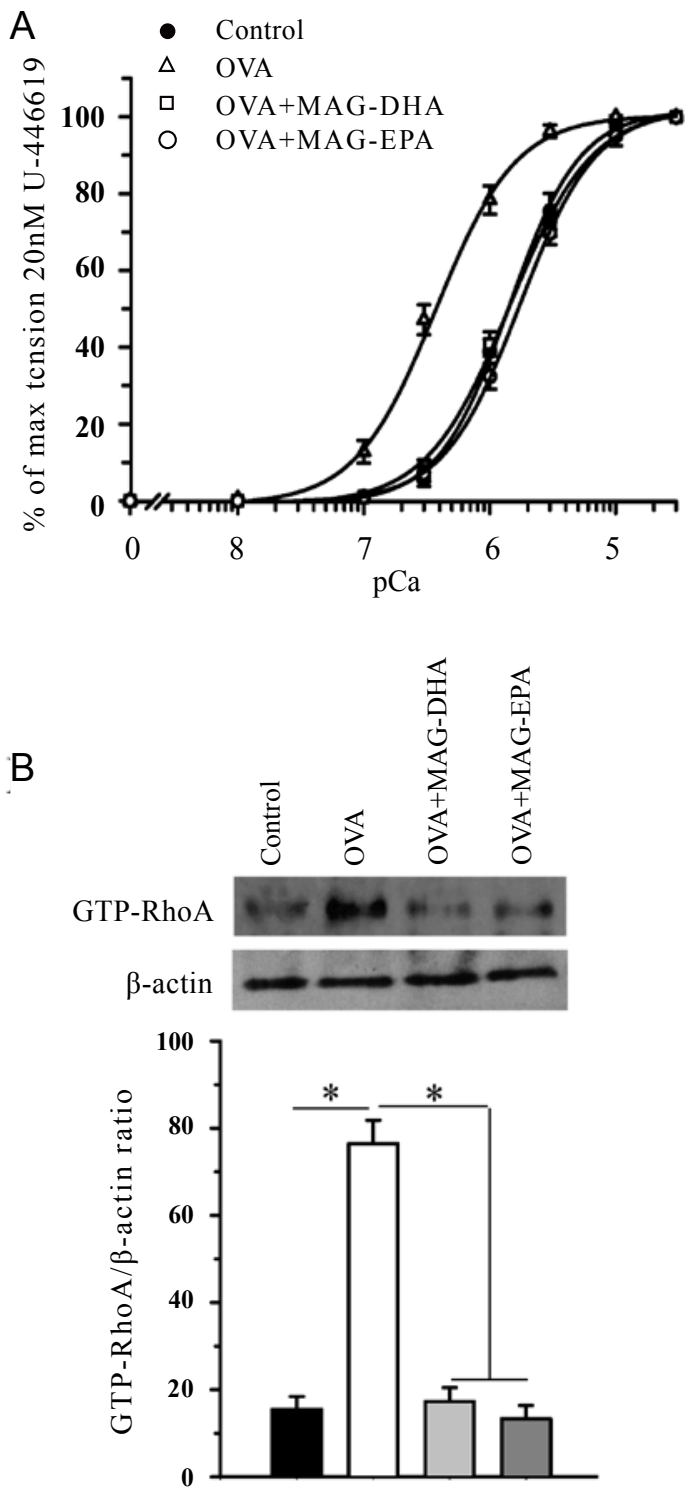

Figure 5: Effect of MAG-DHA and MAG-EPA on U46619-induced $\mathrm{Ca}^{2+}$ hypersensitivity and RhoA activation in OVA sensitized guinea pigs. A: $\mathrm{CCRC}$ to free $\left[\mathrm{Ca}^{2+}\right]$ in the presence of $20 \mathrm{nM} \mathrm{U}-46619$ obtained from $\beta$-escin permeabilized tissues in Control (closed circle, $n=15$ ), OVA (open triangles, $n$ $=20$ ), OVA + MAG-DHA (open squares, $n=18$ ) and OVA + MAG-EPA (open circles, $n=16$ ). Each point represents the mean \pm SEM, ${ }^{*} P<0.05$. B: Rho activation assay was performed on bronchi homogenates derived from Control (untreated), OVA-sensitized, OVA-sensitized + MAG-DHA-treated and OVAsensitized + MAG-EPA-treated guinea pigs. Mean bar histogram displaying the quantitative analysis of various GTP-RhoA density ratios. The $\beta$-actin staining was obtained from the original homogenates derived from the corresponding preparations, $n=5,{ }^{*} p<0.05$. 
effects on inflammatory conditions including asthma [31], and that dietary supplementation with omega-3 fatty acids in children prevents the development of atopic cough, a symptom of allergic airway inflammation [20]. The underlying mechanisms for the beneficial properties of omega-3 fatty acids in asthma remain, however, to be established.

In the present study, we investigated the ability of MAG-DHA and MAG-EPA, newly-synthesized n-3 PUFA monoacylglyceride derivatives, to prevent inflammation and AHR in an in vivo model of allergic asthma. Eosinophils, T-cells and mast cell infiltrates with excess mucus secretion are common features of allergic airway inflammation and have been clinically correlated with AHR [32,33]. Herein, a reduced number of eosinophils and lymphocytes were quantified in BAL fluid recovered from OVA-sensitized guinea pigs receiving a daily per os dose of MAG-DHA or MAG-EPA. Moreover, airway mucus production was lowered in n-3 PUFA monoacylglyceride-treated animals.

In asthma, there is increased expression of multiple inflammatory proteins in the respiratory tract, including cytokines, adhesion molecules, inflammatory enzymes and receptors. The majority of these inflammatory proteins are regulated at the level of gene transcription [29]. NFKB is the main transcription factor involved in up-regulation of inflammatory cytokines, COX2 genes and adhesion molecules [34]. Since airway macrophages and bronchial epithelial cells from asthmatics exhibit increased NFKB activity compared with cells from healthy individuals [34], it has been suggested that NFKB plays a pivotal role in the pathogenesis of asthma [34]. In the present study, we demonstrate that the activation of $\mathrm{NF \kappa B}$ and the over-expression of COX2 inflammatory enzyme are reduced in animals receiving per os MAG-DHA or MAG-EPA treatments. Moreover, in a previous study, we demonstrated that MAG-DHA mediates its anti-inflammatory effects through the activation of the nuclear receptor PPAR $\gamma$ resulting in a decrease in NFKB activation and in related pro-inflammatory gene expression such as cytokines and COX2 in an in vitro model of TNF $\alpha$ stimulated human bronchi [23].

\section{Potential usefulness of n-3 PUFA in regulating RhoA-Rho- kinase pathway in asthma}

Agonist-induced changes in the $\mathrm{Ca}^{2+}$ sensitivity of the contractile apparatus also contribute to pharmaco-mechanical contraction coupling in ASM cells. Evidence has been put forward showing the importance of both PKC and ROCK in this signaling event [7]. Data also suggest that RhoA, a monomeric GTP-binding protein, is a key protein in controlling the tonic response of airway smooth muscles [35]. To explain the profound change seen in AHR which is a hallmark of asthma, there is growing evidence is accumulating for increased activation of the Rho/ROCK signaling pathway following allergen challenge [35]. In a rat model of acute allergen exposure [8], described an increase in acetylcholine-induced $\mathrm{Ca}^{2+}$-independent contractions in rat bronchial smooth muscle which was abolished by treatment with clostridium botulinum C3 exoenzyme (an inhibitor of Rho proteins). Furthermore, molecular biological investigations revealed an upregulation of RhoA and $G_{12,13}$ proteins [36,37], as well as an increase in acetylcholine-induced activation of RhoA [8]. Tumor necrosis factor alpha (a proinflammatory mediator) has been associated with the induction of AHR $[23,38,39]$ and may be responsible for these changes, since it was demonstrated that TNF $\alpha$ enhances the expression and activation of RhoA in ASM [40,41]. Other molecules including IL13 and IL- 4 have been linked to AHR [42]. These evidences suggest that agents able to decrease RhoA activation, could be useful and of clinical interest for prevention and treatment of airway hyperresponsiveness. The usefulness of such agents is supported by the present findings in which newly synthesized n-3 PUFA monoacylglycerides prevented U46619-induced $\mathrm{Ca}^{2+}$ hypersensitivity in OVA-sensitized guinea pigs through the inhibition of RhoA activation. The PKC / CPI-17 pathway, another $\mathrm{Ca}^{2+}$ sensitizing mechanism, has also been demonstrated to be involved under pathophysiological conditions. Several studies show that CPI-17 activation plays a critical role in bronchial reactivity [43-46]. In a previous study, we demonstrated that pro-inflammatory cytokine treatments were able to activate the PKC / CPI-17 pathway leading to an enhanced $\mathrm{Ca}^{2+}$ signaling in human bronchi [43] Moreover, increase expression and activation of CPI-17 were also observed in lung biopsies derived from asthmatic patients [23]. In addition, we also previously demonstrated in a previous study that oral MAG-DHA administration decreased both activation and expression of CPI-17 leading to decreased $\mathrm{Ca}^{2+}$-sensitivity and AHR in a guinea pig OVA-sensitized model of asthma [23]. Hence it is proposed that, in human lung with pathological conditions, the use of MAG-DHA and MAG-EPA would likely decrease $\mathrm{Ca}^{2+}$ hypersensitivity and would be relevant in improving respiratory capacities and recovered pulmonary compliance in asthmatic patients.

\section{Enhanced role of n-3 PUFA in pulmonary pathophysiology}

Clinical assessment of dietary supplementation of omega-3 (n3) polyunsaturated fatty acids (PUFA), including EPA and DHA, has shown their beneficial impact in a wide range of inflammatory diseases [21]. The mode of action of EPA and DHA derivatives through RhoA/ Rho-kinase inhibition/inactivation would support these independent observations. An alternative explanation for these beneficial effects would be that n-3 PUFA competes with arachidonic acid (AA) for enzymatic conversion by COX, LOX and CYP450 enzymes [11]. This competition, in turn would lead to reduced formation of AA metabolites while PUFA-metabolites originating from DHA and EPA are increased, thus modifying the circulating and intracellular omega-3 / omega- 6 ratio. Recent studies have identified a novel group of DHA-derived trihydroxydocosahexanoic acid mediators termed D-series Resolvins, formed from DHA by a series of reactions involving the 5-lipoxygenase pathway. These mediators appear to exert potent anti-inflammatory actions [16]. Intracellular DHA is metabolized through a series of biochemical steps, several of which involve 5 and 15-lipoxygenases, generating a dihydroxydocosatriene termed ProtectinD1, also a potent anti-inflammatory molecule [47]. Indeed, generation of Resolvins and Protectins could represent important anti-inflammatory compounds in the mode of action of $n-3$ PUFAs in addition to having a potent protective role against inflammatory disorders [48-52]. Moreover, our group has already demonstrated that MAG-DHA is metabolized by lipoxygenases in order to mediate its anti-inflammatory effects and to reduce AHR triggered by TNF $\alpha$ in human bronchi, as shown by the combined treatments of 5- and 15-lypoxygenases inhibitors [23]. In addition, we demonstrated that ResolvinD1 and ProtectinD1 prevent AHR and $\mathrm{Ca}^{2+}$ hypersensitivity primarily through their action in reducing COX2 and CPI-17 over-expression. Moreover, in a murine model of chronic asthma, ProtectinD1 administration prior to aeroallergen challenge was shown to result in a decrease in airway eosinophil and T lymphocyte recruitment as well as in reduced levels of specific proinflammatory mediators, including IL-13, cysteinyl leukotrienes and PGD2 [50]. EPA-derived ResolvinE1 also displays anti-inflammatory effects when administrated in a murine model of allergic asthma [49]. Further investigations using high performance liquid chromatography 
coupled to tandem mass spectrometry (HPLC/MS/MS) could enable to determine which metabolite would be potentially responsible for the intracellular effects of MAG-DHA and MAG-EPA in lung or other tissues.

The main limitation of metabolites such as ResolvinE1, ResolvinD1 and ProtectinD1, however, is their relative instability, thus further justifying the use of biochemical precursors or stable analogues. We therefore propose that n-3 PUFA monoacylglyceride derivatives, possessing anti-inflammatory properties, may resolve inflammation and provide an interesting new approach for asthma. Fatty acids in monoglyceride form are generally recognized as safe and are widely used as emulsifying agents in the food industry. Furthermore, pharmacokinetic experiments, performed on rats treated with an oral dose of either DHA monoglyceride (MAG-DHA), DHA triglycerides (DHA-TG) or DHA ethyl ester (DHA-EE), demonstrate that MAGDHA increases the oral bioavailability of DHA compared to DHA-TG and DHA-EE [22]. Moreover, these n-3 PUFA monoacylglycerides prevent U-46619-induced bronchoconstriction through a decrease in RhoA activation thus leading to a reduction in $\mathrm{Ca}^{2+}$ sensitivity of smooth muscle cells. Since the activation of RhoA/Rho kinase pathway is associated with both acute pulmonary bronchoconstriction and long term airway remodeling, finding a lipid mediator able to reduce the activation of Rho-kinase is clearly of potential clinical interest. Moreover, animal and clinical studies have demonstrated that Rhokinase inhibitors could inhibit signal transductions initiated by many broncho-active drugs $[9,35]$; hence it is possible that MAG-DHA and MAG-EPA may exert broader beneficial effects as compared to single receptor antagonists, largely due to its mode of action on intracellular targets. In this respect, it is proposed that n-3 PUFA monoacylglyceride could lead to the production of bioactive metabolites [23] which represent new and prospective pharmacological compounds of low toxicity and medicinal interest in modulating inflammation and bronchoconstriction in asthma. Hence, the down regulation of RhoA/ Rho-kinase pathway by these compounds might explain other systemic effects induced by $n-3$ PUFA derivatives [23, 24, 27].

\section{Acknowledgments}

The authors thank the members of the pathology laboratory for their technical support. This work was supported in part by a grant from Canadian Institutes of Health Research CIHR: MOP 111-112 and a transition grant from the CRC E-LeBel and FMSS, Université de Sherbrooke.

\section{References}

1. Bahadori K, Doyle-Waters MM, Marra C, Lynd L, Alasaly K, et al. (2009) Economic burden of asthma: A systematic review. BMC Pulm Med 9: 24

2. Bousquet J, Jeffery PK, Busse WW, Johnson M, Vignola AM (2000) Asthma from bronchoconstriction to airways inflammation and remodeling. Am J Respir Crit Care Med 161: 1720-1745.

3. Holgate ST, Polosa R (2008) Treatment strategies for allergy and asthma. Nat Rev Immunol 8: 218-230.

4. Mitzner W, Brown RH (2000) Potential mechanism of hyperresponsive airways Am J Respir Crit Care Med 161: 1619-1623.

5. Janssen LJ (1998) Calcium handling in airway smooth muscle: Mechanisms and therapeutic implications. Can Respir J 5: 491-498.

6. Tliba O, Deshpande D, Chen H, Van Besien C, Kannan M, et al. (2003) IL-13 enhances agonist-evoked calcium signals and contractile responses in airway smooth muscle. Br J Pharmacol 140: 1159-1162.

7. Somlyo AP, Somlyo AV (2003) $\mathrm{Ca}^{2+}$ sensitivity of smooth muscle and nonmuscle myosin II: Modulated by G proteins, kinases, and myosin phosphatase. Physiol Rev 83: 1325-1358.
8. Chiba Y, Takada Y, Miyamoto S, MitsuiSaito M, Karaki H, et al. (1999) Augmented acetylcholine-induced, rho-mediated $\mathrm{Ca}^{2+}$ sensitization of bronchia smooth muscle contraction in antigen-induced airway hyperresponsive rats. $\mathrm{Br}$ J Pharmacol 127: 597-600.

9. Hashimoto K, Peebles RS, Sheller JR, Jarzecka K, Furlong J, et al. (2002) Suppression of airway hyperresponsiveness induced by ovalbumin sensitisation and RSV infection with Y-27632, a rho kinase inhibitor. Thorax 57: 524-527.

10. Babcock TA, Helton WS, Anwar KN, Zhao YY, Espat NJ (2004) Synergistic anti-inflammatory activity of omega-3 lipid and rofecoxib pretreatment on macrophage proinflammatory cytokine production occurs via divergent NFkappaB activation. JPEN J Parenter Enteral Nutr 28: 232-239.

11. Sijben JW, Calder PC (2007) Differential immunomodulation with long-chain n-3 PUFA in health and chronic disease. Proc Nutr Soc 66: 237-259.

12. Corey EJ, Shih C, Cashman JR (1983) Docosahexaenoic acid is a strong inhibitor of prostaglandin but not leukotriene biosynthesis. Proc Natl Acad Sci U S A 80: 3581-3584.

13. Needleman P, Raz A, Minkes MS, Ferrendelli JA, Sprecher H (1979) Triene prostaglandins: Prostacyclin and thromboxane biosynthesis and unique biological properties. Proc Natl Acad Sci U S A 76: 944-948.

14. Lee TH, Mencia-Huerta JM, Shih C, Corey EJ, Lewis RA, et al. (1984) Effects of exogenous arachidonic, eicosapentaenoic, and docosahexaenoic acids on the generation of 5-lipoxygenase pathway products by ionophore-activated human neutrophils. J Clin Invest 74: 1922-1933.

15. Serhan CN, Clish CB, Brannon J, Colgan SP, Chiang N, et al. (2000) Nove functional sets of lipid-derived mediators with antiinflammatory actions generated from omega-3 fatty acids via cyclooxygenase 2-nonsteroidal antiinflammatory drugs and transcellular processing. J Exp Med 192: 11971204.

16. Serhan CN, Hong S, Gronert K, Colgan SP, Devchand PR, et al. (2002) Resolvins: A family of bioactive products of omega-3 fatty acid transformation circuits initiated by aspirin treatment that counter proinflammation signals. J Exp Med 196: 1025-1037.

17. Hong S, Gronert K, Devchand PR, Moussignac RL, Serhan CN (2003) Nove docosatrienes and 17S-resolvins generated from docosahexaenoic acid in murine brain, human blood, and glial cells. Autacoids in anti-inflammation. $J$ Biol Chem 278: 14677-14687.

18. Jia Q, Lupton JR, Smith R, Weeks BR, Callaway E, et al. (2008) Reduced colitis-associated colon cancer in fat- 1 ( $\mathrm{n}-3$ fatty acid desaturase) transgenic mice. Cancer Res 68: 3985-3991.

19. Freedman SD, Blanco PG, Zaman MM, Shea JC, Ollero M, et al. (2004) Association of cystic fibrosis with abnormalities in fatty acid metabolism. N Eng J Med 350: 560-569.

20. Peat JK, Mihrshahi S, Kemp AS, Marks GB, Tovey ER, et al. (2004) Three-yea outcomes of dietary fatty acid modification and house dust mite reduction in the childhood asthma prevention study. J Allergy Clin Immunol 114: 807-813.

21. Connor WE (2000) Importance of $n-3$ fatty acids in health and disease. Am J Clin Nutr 71: 171S-175S.

22. Fortin S (2009) Polyunsaturated fatty acid monoglycerides, derivatives, and uses thereof. Can Patent 2672513

23. Morin C, Fortin S, Cantin AM, Rousseau E (2011) Docosahexaenoic acid derivative prevents inflammation and hyperreactivity in lung: Implication of PKC potentiated inhibitory protein for heterotrimeric myosin light chain phosphatase of $17 \mathrm{kD}$ in asthma. Am J Respir Cell Mol Biol 45: 366-375.

24. Morin C, Fortin S, Guibert C, Rousseau E (2011) $\omega 3$ and $\omega 6$ CYP450 eicosanoid derivatives: key lipid mediators in the regulation of pulmonary hypertension. Book chapter in Pulmonary Hypertension - From Bench Research to Clinical Challenges.

25. Reagan-Shaw S, Nihal M, Ahmad N (2008) Dose translation from animal to human studies revisited. FASEB J 22: 659-661.

26. Morin C, Fortin S, Rousseau E (2011) Bronchial inflammation induced PKC over-expression: involvement in mechanical properties of airway smooth muscle. Can J Physiol Pharmacol, in press.

27. Morin C, Fortin S, Rousseau E (2011) 19,20-EpDPE, a bioactive CYP450 metabolite of DHA monoacyglyceride, decreases $\mathrm{Ca}^{2+}$ sensitivity in human pulmonary arteries. Am J Physiol Heart Circ Physiol 301: H1311-1318. 
Citation: Morin C, Fortin S, Rousseau É (2012) New Omega-3 Derivatives Reduce Airway Inflammation and Prevent Rho-Kinase Activation in an Allergic Model of Asthma. J Aller Ther S1:003. doi:10.4172/2155-6121.S1-003

28. Gagliardo R, Chanez P, Mathieu M, Bruno A, Costanzo G, et al. (2003) Persistent activation of nuclear factor-kappaB signaling pathway in severe uncontrolled asthma. Am J Respir Crit Care Med 168: 1190-1198.

29. Murdoch JR, Lloyd CM (2009) Chronic inflammation and asthma. Mutat Res 690: 24-39.

30. Hudert CA, Weylandt KH, Lu Y, Wang J, Hong S, et al. (2006) Transgenic mice rich in endogenous omega-3 fatty acids are protected from colitis. Proc Nat Acad Sci U S A 103: 11276-11281.

31. Schwartz J, Weiss ST (1994) The relationship of dietary fish intake to level of pulmonary function in the first national health and nutrition survey (NHANES I). Eur Respir J 7: 1821-1824.

32. Siddiqui S, Hollins F, Saha S, Brightling CE (2007) Inflammatory cell microlocalisation and airway dysfunction: Cause and effect? Eur Respir J 30: 1043-1056.

33. Kearley J, Buckland KF, Mathie SA, Lloyd CM (2009) Resolution of allergic inflammation and airway hyperreactivity is dependent upon disruption of the T1 ST2-IL-33 pathway. Am J Respir Crit Care Med 179: 772-781.

34. Hart LA, Krishnan VL, Adcock IM, Barnes PJ, Chung KF (1998) Activation and localization of transcription factor, nuclear factor-kappaB, in asthma. Am J Respir Crit Care Med 158: 1585-1592.

35. Chiba Y, Misawa M (2004) The role of RhoA-mediated $\mathrm{Ca}^{2+}$ sensitization of bronchial smooth muscle contraction in airway hyperresponsiveness. J Smooth Muscle Res 40: 155-167.

36. Chiba Y, Sakai H, Wachi H, Sugitani H, Seyama Y, et al. (2003) Upregulation of rhoA mRNA in bronchial smooth muscle of antigen-induced airway hyperresponsive rats. J Smooth Muscle Res 39: 221-228.

37. Chiba $Y$, Misawa $M$ (2001) Increased expression of $G_{12}$ and $G_{13}$ proteins in bronchial smooth muscle of airway hyperresponsive rats. Inflamm Res 50: 333336.

38. Anticevich SZ, Hughes JM, Black JL, Armour CL (1995) Induction of human airway hyperresponsiveness by tumour necrosis factor-alpha. Eur J Pharmacol 284: 221-225.

39. Sukkar MB, Hughes JM, Armour CL, Johnson PR (2001) Tumour necrosis factor-alpha potentiates contraction of human bronchus in vitro. Respirology 6: 199-203.

40. Sakai H, Otogoto S, Chiba Y, Abe K, Misawa M (2004) TNF-alpha augments the expression of RhoA in the rat bronchus. J Smooth Muscle Res 40: 25-34.
41. Hunter I, Cobban HJ, Vandenabeele P, MacEwan DJ, Nixon GF (2003) Tumor necrosis factor-alpha-induced activation of RhoA in airway smooth muscle cells: Role in the $\mathrm{Ca}^{2+}$ sensitization of myosin light chain20 phosphorylation. Mol Pharmacol 63: 714-721.

42. Chiba Y, Nakazawa S, Todoroki M, Shinozaki K, Sakai H, et al. (2009) Interleukin-13 augments bronchial smooth muscle contractility with an upregulation of RhoA protein. Am J Respir Cell Mol Biol 40: 159-167.

43. Morin C, Sirois M, Echave V, Rousseau E (2008) CPI-17 silencing-reduced responsiveness in control and TNF-alpha-treated human bronchi. Am J Respir Cell Mol Biol 39: 638-643.

44. Morin C, Sirois M, Echave V, Gomes MM, Rousseau E (2007) Epoxyeicosatrienoic acid relaxing effects involve $\mathrm{Ca}^{2+}$-activated $\mathrm{K}^{+}$channe activation and $\mathrm{CPI}-17$ dephosphorylation in human bronchi. Am J Respir Cell Mol Biol 36: 633-641.

45. Sakai H, Chiba Y, Hirano T, Misawa M (2005) Possible involvement of CPI-17 in augmented bronchial smooth muscle contraction in antigen-induced airway hyper-responsive rats. Mol Pharmacol 68: 145-151.

46. Sakai H, Hirano T, Chiba Y, Misawa M (2005) Acetylcholine-induced phosphorylation and membrane translocation of $\mathrm{CPI}-17$ in bronchial smooth muscle of rats. Am J Physiol Lung Cell Mol Physiol 289: L925-930.

47. Serhan CN, Gotlinger K, Hong S, Lu Y, Siegelman J, et al. (2006) Antiinflammatory actions of neuroprotectin D1/protectin D1 and its natura stereoisomers: Assignments of dihydroxy-containing docosatrienes. J Immunol 176: $1848-1859$

48. Kohli P, Levy BD (2009) Resolvins and protectins: Mediating solutions to inflammation. $\mathrm{Br} \mathrm{J}$ Pharmacol 158: 960-971.

49. Aoki H, Hisada T, Ishizuka T, Utsugi M, Kawata T, et al. (2008) Resolvin E1 dampens airway inflammation and hyperresponsiveness in a murine model of asthma. Biochem Biophys Res Commun 367: 509-515.

50. Levy BD, Kohli P, Gotlinger K, Haworth O, Hong S, et al. (2007) Protectin D1 is generated in asthma and dampens airway inflammation and hyperresponsiveness. J Immunol 178: 496-502.

51. Lukiw WJ, Cui JG, Marcheselli VL, Bodker M, Botkjaer A, et al. (2005) A role for docosahexaenoic acid-derived neuroprotectin D1 in neural cell survival and alzheimer disease. J Clin Invest 115: 2774-2783.

52. Marcheselli VL, Hong S, Lukiw WJ, Tian XH, Gronert K, et al. (2003) Nove docosanoids inhibit brain ischemia-reperfusion-mediated leukocyte infiltration and pro-inflammatory gene expression. J Biol Chem 278: 43807-43817.
This article was originally published in a special issue, Airway Inflammation and Hyper responsiveness handled by Editor(s). Dr. John F. Alcorn (1), Children's Hospital of Pittsburgh, USA. 